\section{Ocular angiostrongyliasis: clinical study of three cases}

\begin{abstract}
Purpose To report three patients with ocular angiostrongyliasis who presented with a variety of clinical findings.

Method Retrospective, observational case series. The medical charts, photographs, and electrophysiologic records were reviewed.

Results All patients presented with blurred vision and one had a history of eosinophilic meningitis. In each respective case, only one living larva was found in the anterior chamber, vitreous cavity, and subretinal space. The fundus examination revealed generalized retinal pigment epithelial alteration, subretinal tracks, retinal oedema, macular oedema, and a pale disc. Visually evoked potentials showed delayed latency time in one patient, which represented the secondary optic neuritis. Blood eosinophilia was not detected, and stool examinations did not show

Angiostrongylus cantonensis larva or its egg. In both cases of surgical removal, an immature male worm was identified by the parasitologist. After treatment, the visual acuity was slightly improved in all cases. Conclusion This case series illustrated the different ocular manifestations of angiostrongyliasis and that although several treatments were used, the visual outcome was not markedly improved and depended mainly on the initial visual acuity.

Eye (2008) 22, 1446-1448; doi:10.1038/eye.2008.135; published online 6 June 2008
\end{abstract}

Keywords: Angiostrongylus cantonensis; intraocular; ocular angiostrongyliasis

\section{Introduction}

Ocular manifestation is a very rare event of angiostrongyliasis, but because of eating habit, it does occur in some developing areas of the
S Sinawat ${ }^{1}$, T Sanguansak¹, T Angkawinijwong ${ }^{1}$, T Ratanapakorn ${ }^{1}$, PM. Intapan², S Sinawat ${ }^{3}$ and Y Yospaiboon ${ }^{1}$ world. Evidently, less than 20 cases have been previously reported from Thailand, Vietnam, Japan, Taiwan, Papua New Guinea, India, and Sri Lanka. ${ }^{1-7}$ We herein report three cases of ocular angiostrongyliasis, the parasites being found in a different part of each patient's eye. These ocular findings will provide the useful clinical information.

\section{Case reports}

Case 1

A 47-year-old man presented having had blurred vision in the left eye for 3 weeks. He had a history of eating raw food, but he denied a history of headache. During the ocular examination, he could count finger at $2 \mathrm{ft}$ with the affected eye. A parasite was found in the anterior chamber without intraocular inflammation (Figure 1a). Relative afferent papillary defect (RAPD) was positive on the left side. The fundus examination showed generalized retinal pigment epithelial alteration, retinal haemorrhage at superotemporal area, and normal disc appearance (Figure 2). Visually evoked potentials (VEP) showed delayed latency time with normal amplitude. Serum was positive for $29 \mathrm{kDa}$ for Angiostrongylus cantonensis antibody. Sarcocystic spp. oocyst and Opisthorchis viverrini egg were found during the stool examination. An immature male worm was aspirated by simcoe cannula after laser photocoagulation. Intravenous methylprednisolone injection was prescribed for secondary optic neuritis. The final visual acuity was $2 / 60$.

Case 2

A 27-year-old man presented with progressive visual loss in the left eye for 3 weeks. He reported a history of eosinophilic meningitis 2 months before the onset of blurred vision. He 

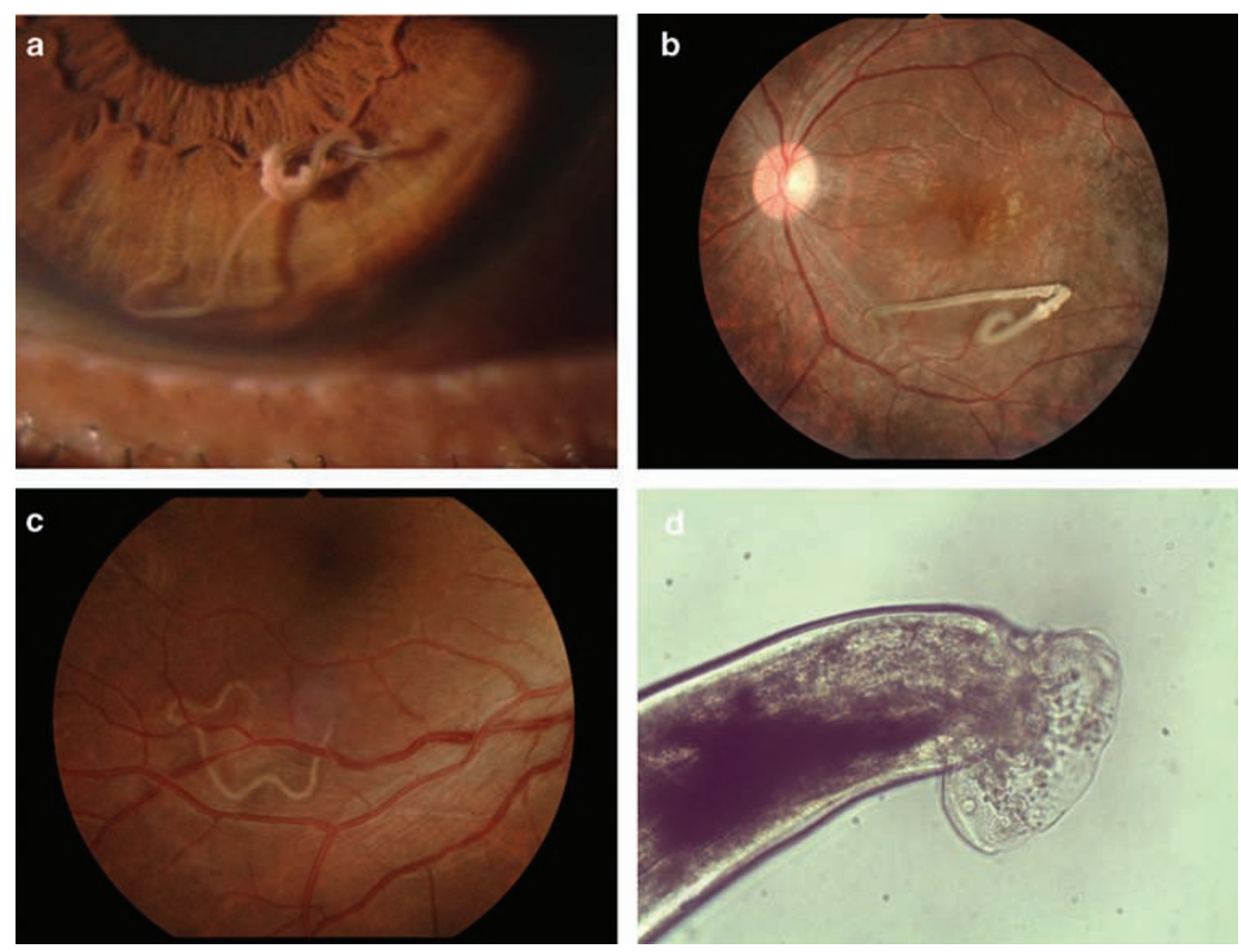

Figure 1 Three cases of ocular angiostrongyliasis. (a) Intracameral parasite. (b) Intravitreal parasite. (c) Subretinal parasite. (d) Bursa at the posterior structure of a male larva.

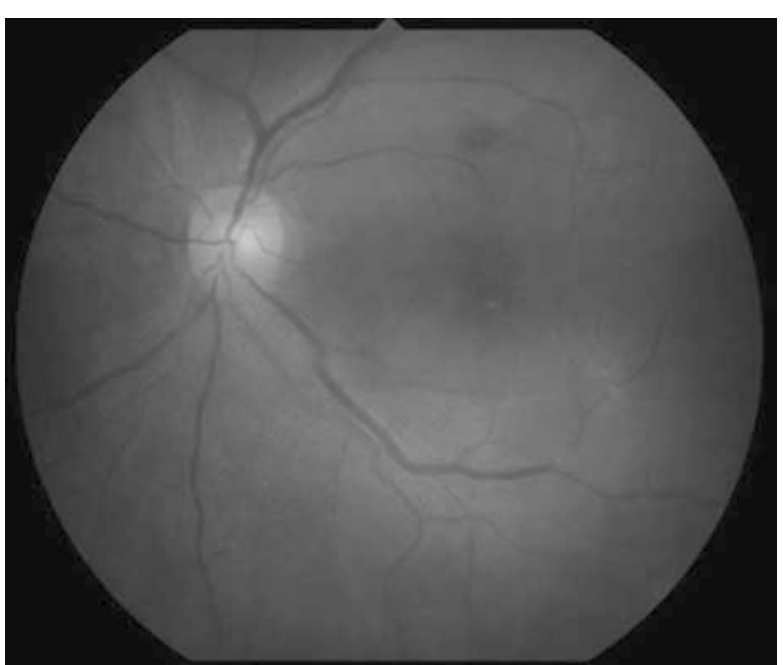

Figure 2 Generalized retinal pigment epithelial alteration, retinal haemorrhage at superotemporal area and pigment epithelial detachment next to the foveal centre were induced by Angiostrongylus cantonensis.

also had a history of ingestion of raw Pila spp. snails. Initial visual acuity was $1 / 60$ with RAPD positive on the left side. Cell trace and flare trace were detected in the anterior vitreous. The fundus examination showed multiple subretinal tracks, a rather pale disc, and generalized retinal pigment epithelial alteration. An intravitreal movable larva was found in the superotemporal area. Diode laser was directly applied to the parasite until it was dead in the inferotemporal area (Figure 1b). The pars plana vitrectomy and parasite removal were successfully performed, and then an immature male worm was identified by a parasitologist. There were minimal intraocular inflammations in the post-operative period; thus he was treated with topical steroid. His vision was slightly improved to $6 / 60$.

\section{Case 3}

A 36-year-old man developed visual loss in his right eye for 1 week. He denied any history of headache. He took mebendazole orally two to three times per year; notwithstanding, a Tenia spp. worm was found in his stool. Visual acuity of the affected eye was $2 / 60$. The intraocular inflammation was not detected and the RAPD was negative. A subretinal living parasite with subretinal tracks at superotemporal fundus and macular oedema were detected by indirect ophthalmoscope (Figure 1c). The electroretinogram and VEP were normal. The parasite was treated with a diode laser. Topical prednisolone acetate was prescribed to reduce the subsequent inflammation. An Echinostoma egg was found on stool examination. His final vision was 6/60. 


\section{Discussion}

Only $1.1 \%$ of angiostrongyliasis had ocular manifestation, ${ }^{8}$ which is diagnosed by identification of A. cantonensis in any part of the eye. People are usually infected by eating the intermediate host raw, especially Pila spp. snails. The incubation period is then between 2 weeks and 2 months. The most common symptom is blurred vision without headache although the eosinophilic meningitis may be presented prior to, or co-existing with, the ocular symptoms. The parasite is commonly found in the vitreous cavity.

In our case series, only one patient had a history of eosinophilic meningitis. As with previous reports, only one larva was detected in each case and all worms were still alive. The parasites were easily identified and distinguished from other parasites by indirect ophthalmoscope. In the case of surgical removal, an immature male worm was clearly identified by bursa, the posterior structures of the larva.

Many ocular findings have been reported in previous studies including uveitis, subretinal tracks, necrotizing retinitis, disc swelling, retinal pigment alteration, and retinal detachment. ${ }^{9-12}$ Although blood eosinophilia and the antibody response are demonstrated in most cases of eosinophilic meningitis, it has not been observed in ocular angiostrongyliasis without meningitis. In our series, an antibody response in the patient without eosinophilic meningitis was shown.

Larva and eggs were seldom found on stool examination. Several treatments were used, including intravenous methylprednisolone, topical prednisolone, laser and surgical removal. Unfortunately, the therapy did not dramatically improve visual function, which was mainly dependent on initial visual acuity.

\section{References}

1 Chiu JK, Huang WH, Cheng KH, Chang IH, Teng WH, Kao TH. Three cases of worm proven human angiostrongyliasis in Taiwan. Zhonghua Min Guo Wei Sheng Wu Ji Mian Yi Xue Za Zhi 1981; 14: 247-250.

2 Scrimgeour EM, Chambers BR, Kaven J. A probable case of ocular angiostrongyliasis in New Britain, Papua New Guinea. Trans R Soc Trop Med Hyg 1982; 76: 538-540.

3 Singalavanija A, Wangspa S, Teschareon S. Intravitreal angiostrongyliasis. Aust N Z J Ophthalmol 1986; 14: 381-384.

4 Durette-Desset MC, Chabaud AG, Cassim MH, Ismail MM, Premaratne UN, Abeyewickreme $\mathrm{W}$ et al. On an infection of a human eye with Parastrongylus (=Angiostrongylus) sp. in Sri Lanka. J Helminthol 1993; 67: 69-72.

5 Toma H, Matsumura S, Oshiro C, Hidaka T, Sato Y. Ocular angiostrongyliasis without meningitis symptoms in Okinawa, Japan. J Parasitol 2002; 88: 211-213.

6 Thu TP, Nguyen NX, Lan le T, Kuchle M. Ocular Angiostrongylus cantonensis in a female Vietnamese patient: case report. Klin Monatsbl Augenheilkd 2002; 219: 892-895.

7 Patikulsila D, Ittipunkul N, Theerakittikul B. Intravitreal angiostrongyliasis: report of 2 cases. J Med Assoc Thai 2003; 86: $981-985$.

8 Sawanyawisuth K, Kitthaweesin K, Limpawattana P, Intapan PM, Tiamkao S, Jitpimolmard S et al. Intraocular angiostrongyliasis: clinical findings, treatments and outcomes. Trans R Soc Trop Med Hyg 2007; 101: 497-501.

9 Kumar V, Kyprianou I, Keenan JM. Ocular Angiostrongyliasis: removal of a live nematode from the anterior chamber. Eye 2005; 19: 229-230.

10 Wang LC, Wang IH, Jou JR. Optic neuritis secondary to Angiostrongylus cantonensis infection. Ocul Immunol Inflamm 2006; 14(3): 189-191.

11 Liu IH, Chung YM, Chen SJ, Cho WL. Necrotizing retinitis induced by Angiostrongylus cantonensis. Am J Ophthalmol 2006; 141: 577-579.

12 Malhotra S, Mehta DK, Arora R, Chauhan D, Ray S, Jain M. Ocular angiostrongyliasis in a child - first case report from India. J Trop Pediatr 2006; 52: 223-225. 